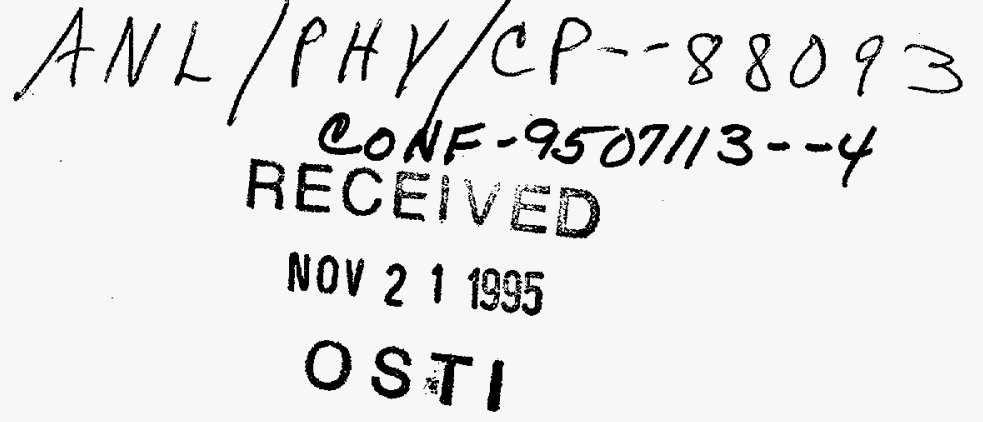

\title{
Separable approximation to the Bethe-Salpeter equation in QCD
}

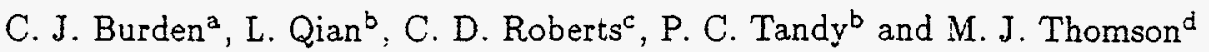 \\ ${ }^{a}$ Department of Theoretical Physics, Research School of Physical Sciences and Engineering, \\ Australian National University, Canberra, ACT 0200, Australia
}

${ }^{b}$ Department of Physics, Kent State University, Kent, Ohio, USA 44242

'Physics Division, Argonne National Laboratory, Argonne, Illinois, USA 60439

${ }^{d}$ School of Physics, University of Melbourne, Parkville, Victoria 3052, Australia

We calculate the mass spectrum and decay constants of the pseudoscalar meson octet, and mass spectrum of the vector meson octet from a separable approximation to the Bethe-Salpeter equation. In the model, quark propagators inspired by earlier studies of QCD Dyson-Schwinger equations are taken as the only input.

\section{INTRODUCTION}

While lattice gauge theory provides the closest available realisation of an exact non-perturbative treatment of $\mathrm{QCD}$, much can also be learnt from QCD based models of hadronic physics. A most promising line of attack in this direction is the application of Dyson-Schwinger (DS) and BetheSalpeter (BS) equations [1] to calculations of hadronic properties, including hadron masses, decay constants and form factors. The philosophy behind these calculations is one of attempting to understand physical processes driving observed phenomena, rather than numerical verification of QCD itself. As such they provide an invaluable complement to the work of lattice gauge theorists.

The dynamics of the light mesons is a sensitive test of confinement and dynamical chiral symmetry breaking in $Q C D$, with the pion being realised as an almost-Goldstone boson. Central to the success of the model described below is the fact that the combined rainbow DS equation and homogeneous ladder BS equation have the property that, in the chiral limit, they admit as solutions massless Goldstone pions [2]. This property has been used to advantage in a number of earlier studies [3] of light mesons. We present here a model of the light scalar and vector mesons differing from previous models in that the only inputs are light quark propagators inspired by es- tablished studies of QCD DS equations $[1,4,5]$. In this, and in a parallel study [6], the BetheSalpeter kernel is approximated by a separable effective gluon propagator obtained by inverting the quark DS equation. It is of interest to see whether this simple gluon propagator, endowed with just the Goldstone knowledge of the quark propagator, can also produce good results for the non-Goldstone vector mesons. More complete details of this study will be presented elsewhere [7].

\section{THE MODEL}

We begin with the combination of a rainbow approximation DS equation for each quark propagator [1],

$$
\begin{aligned}
& S_{f}^{-1}(p)-i \not p-m= \\
& \quad \frac{4}{3} \int \frac{d^{4} q}{(2 \pi)^{4}} \gamma_{\mu} S_{f}(q) D(p-q) \gamma_{i \mu},
\end{aligned}
$$

and the homogeneous ladder approximation to the meson Bethe-Salpeter equation,

$$
\begin{aligned}
& \Gamma(p, P)=-\frac{4}{3} \int \frac{d^{4} q}{(2 \pi)^{4}} D(p-q) \times \\
& \quad \gamma_{\mu} S_{f_{3}}(q-\xi P) \Gamma(q, P) S_{f_{2}}(q+(1-\xi) P) \gamma_{\mu} .
\end{aligned}
$$

Here $S_{f}(p)$ is the full quark propagator of flavour $f, \delta_{\mu \nu} D(p-q)$ the Feynman gauge effective gluon propagator and $\Gamma(q, P)$ the BS amplitude defined 
so that external outgoing and incoming quark lines carry momenta $q+(1-\xi) P$ and $q-\xi P$ respectively. By rainbow approximation we mean that the full quark-gluon vertex has been replaced by the bare vertex. The metric is Euclidean.

The most general form of the fermion propagators consistent with Lorentz covariance, including $\mathrm{C}, \mathrm{P}$ and $\mathrm{T}$, is given in terms of two scalar functions. Here we use either of the two following representations for our quark propagators:

$$
\begin{aligned}
S(p) . & =-i \not p \sigma_{V}\left(p^{2}\right)+\sigma_{S}\left(p^{2}\right) \\
& =\frac{1}{i \not p A\left(p^{2}\right)+B\left(p^{2}\right)} .
\end{aligned}
$$

The signature of a confined particle is one whose propagator has no timelike poles [4]. To ensure quark confinement within the model, $\sigma_{V}$ and $\sigma_{S}$ are assumed to be entire functions in the complex $p^{2}$ plane. It is these two functions which provide the only free input to the model. A separable approximation to the Feynman gauge gluon propagator is obtained by assuming a form

$D(p-q)=G\left(p^{2}\right) G\left(q^{2}\right)+p \cdot q F\left(p^{2}\right) F\left(q^{2}\right)$.

Eq. (1) is then solved by the ansatz

$F\left(q^{2}\right)=\frac{1}{a}\left(A\left(q^{2}\right)-1\right)$,
$G\left(q^{2}\right)=\frac{1}{b}\left(B\left(q^{2}\right)-m\right)$,

where $a$ and $b$ are given by

$$
\begin{aligned}
& a^{2}=\frac{2}{3} g^{2} \int \frac{d^{4} q}{(2 \pi)^{4}} q^{2} \sigma_{V}\left(q^{2}\right)\left(A\left(q^{2}\right)-1\right), \\
& b^{2}=\frac{16}{3} g^{2} \int \frac{d^{4} q}{(2 \pi)^{4}} \sigma_{S}\left(q^{2}\right)\left(B\left(q^{2}\right)-m\right) .
\end{aligned}
$$

For the case of unequal constituent quark masses (e.g. the Kaon BS equation), a separable approximation to the the gluon propagator is formed by taking a symmetric combination of the functions $F$ and $G$ obtained for the individual quarks in such a way that Eq. (4) is recovered in the limit $m_{2} \rightarrow m_{1}$.

\section{METHOD OF SOLUTION}

We give here a brief description of the method of solution of the model to determine the meson masses and decay constants. The basic idea is straightforward. Once the quark and effective gluon propagators are specified by Eqs. (3), (4) and (5), the BS equation (2) is a linear integral equation for the amplitude $\Gamma$. This equation is solved on mass-shell by setting the meson Euclidean 4-momentum equal to

$P_{\mu}=(0, i M)$,

and adjusting the bound state mass $M$ until the linear integral operator defined by the right hand side of Eq. (2) has an eigenvalue of 1.

The specific details depend on the meson in question. Consider, for instance, the case of a pseudoscalar meson for which the BS amplitude must take the general form [8]

$$
\begin{aligned}
\Gamma^{\text {pseud }}(p, P)=\gamma_{5}\left\{g_{1}\left(p^{2}, P^{2}, p \cdot P\right)+\right. \\
\\
\quad P g_{2}\left(p^{2}, P^{2}, p \cdot P\right)+\not p g_{3}\left(p^{2}, P^{2}, p \cdot P\right)+ \\
\left.\quad[P, p] g_{4}\left(p^{2}, P^{2}, p \cdot P\right)\right\} .
\end{aligned}
$$

With $P_{\mu}$ as in Eq. (8), the scalar functions $g_{\text {; }}$ are functions of $p_{4}$ and $|\mathbf{p}|$. Furthermore, since the only $p$-dependence on the right hand side of Eq. (2) arises from $D(p-q)$, we see that, in the separable approximation Eq. (4), the $g_{i}$ must be a linear combination of $G\left(p^{2}\right), p_{4} F\left(p^{2}\right)$ and $|\mathbf{p}| F\left(p^{2}\right)$ :

$$
\begin{aligned}
& g_{1}(p)=\lambda_{1} G\left(p^{2}\right)+\lambda_{2} p_{4} F\left(p^{2}\right)+\lambda_{3}|\mathrm{p}| F\left(p^{2}\right), \\
& g_{2}(p)=\lambda_{4} G\left(p^{2}\right)+\lambda_{5} p_{4} F\left(p^{2}\right)+\lambda_{6}|\mathrm{p}| F\left(p^{2}\right), \\
& g_{3}(p)=\lambda_{7} G\left(p^{2}\right)+\ldots
\end{aligned}
$$

By substitution into Eq. (2) and carrying out some straightforward Dirac algebra one obtains a matrix equation of the form

$$
\left(\begin{array}{c}
\lambda_{1} \\
\lambda_{2} \\
\lambda_{3} \\
\vdots
\end{array}\right)=K(M)\left(\begin{array}{c}
\lambda_{1} \\
\lambda_{2} \\
\lambda_{3} \\
\vdots
\end{array}\right),
$$

where

$$
\begin{aligned}
& K(M)= \\
& \quad\left(\begin{array}{ccc}
\int d q G T_{11} G & \int d q q_{4} F T_{12} G & \ldots \\
\int d q q_{4} F T_{21} G & \int d q q_{4} F T_{12} q_{4} F & \ldots \\
\int d q|q| F T_{12} G & \ldots & \\
\vdots & &
\end{array}\right)
\end{aligned}
$$


and the $T_{i j}$ depend on $q_{4},|q|$, and $M$. The matrix $K(M)$ can be evaluated numerically with moderate amounts of computer time, and the meson mass $M$ adjusted until an eigenvalue of 1 is obtained.

The pseudoscalar decay constants are determined entirely by mass shell BS amplitudes. From the definitions of the decay constant, quark propagators and BS amplitude, one obtains [1]

$$
\begin{aligned}
& P^{2} f_{P}=\frac{N_{\epsilon}}{\sqrt{ } 2} \int \frac{d^{4} k}{(2 \pi)^{4}} \operatorname{tr}\left[P_{\gamma_{5}} \times\right. \\
& \left.S_{f_{1}}(p-\xi P) \Gamma(p, P) S_{f_{3}}(p+(1-\xi) P)\right],
\end{aligned}
$$

which enables a direct evaluation of $f_{P}$ once the BS amplitude has been correctly normalised [8].

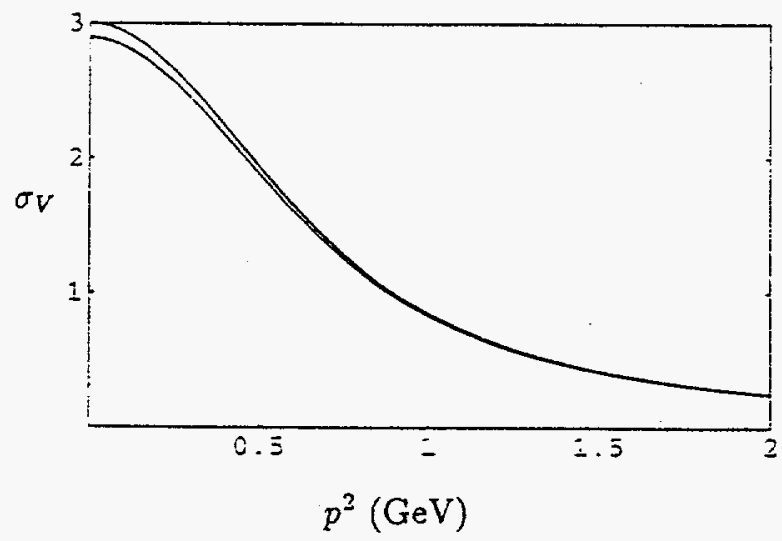

Figure 1. The analytic function $\sigma_{V}\left(p^{2}\right)$ in the quark propagator for the up/down quark (upper curve) and the strange quark (lower curve).

\section{RESULTS}

The input propagator functions $\sigma_{V}\left(p^{2}\right)$ and $\sigma_{S}\left(p^{2}\right)$ for up, down and strange quarks are plotted in Figures 1 and 2. The up and down quarks are assumed degenerate for the purposes of the model. $\sigma_{V}$ and $\sigma_{S}$ are entire functions with analytic forms arising from analyses of approximate DS equations [4]. These forms have been success-

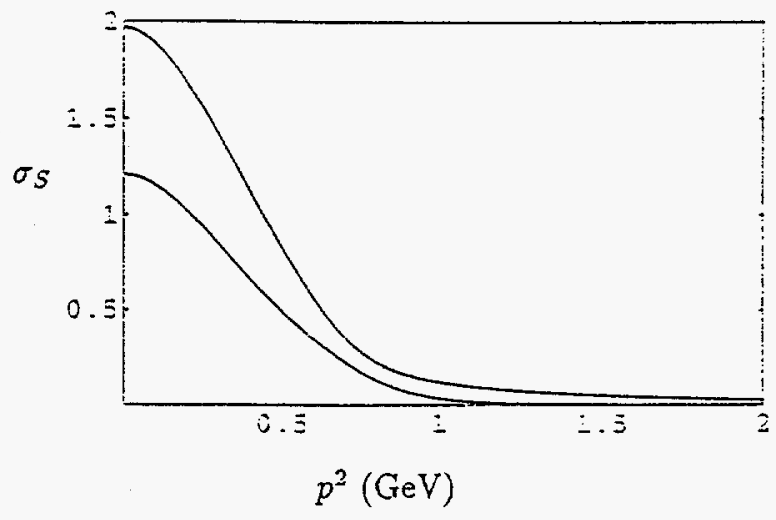

Figure 2. The analytic function $\sigma_{S}\left(p^{2}\right)$ in the quark propagator for the up/down quark (lower curve) and the strange quark (upper curve).

fully applied elsewhere to studies of the electromagnetic pion and kaon form factors [9]. In order to ensure convergence of the integrals in Eqs. (6) and ( 7$)$, we must have

$\sigma_{V}\left(p^{2}\right)=\frac{1}{p^{2}+m^{2}}+o\left(p^{-4}\right)$
$\sigma_{S}\left(p^{2}\right)=\frac{m}{p^{2}+m^{2}}+o\left(p^{-4}\right)$.

This is achieved by the introduction of an ultraviolet regulator in the form of gaussian damping functions incorporated into $\sigma_{V}$ and $\sigma_{S}$. The freedom allowed in setting the chiral quark masses and the ultraviolet regulator enables a fit to the masses and decay constants of the pion and kaon.

Our preliminary results are shown in Table 1 for light scalar, pseudoscalar and vector mesons. For the eta, calculations were carried out assuming octet-singlet mixing angles (defined by $|\eta\rangle=\cos \theta|8\rangle-\sin \theta|1\rangle)$ of $\theta=0^{\circ}$ and $5^{\circ}$. The results for $m_{\eta}$ favour a mixing angle of $5^{\circ}$. The results are in good agreement with experiment for all states with the exception of the scalar mesons which come out well below the observed lightest scalar mesons $a_{0}(980), f_{0}(975)$ and $K_{0}^{*}(1430)$. The general consensus in the literature regard- 
Table 1

Meson results

\begin{tabular}{lcr}
\hline & $\begin{array}{c}\text { Calculation } \\
(\mathrm{MeV})\end{array}$ & $\begin{array}{r}\text { Experiment [10] } \\
(\mathrm{MeV})\end{array}$ \\
\hline$m_{\pi}$ & 137.5 (fit) & $\pi^{ \pm}(140), \pi^{0}(135)$ \\
$f_{\pi}$ & 92.4 (fit) & $\pi^{+}(92.4)$ \\
$m_{\rho / \omega}$ & 754.8 & $\omega(783), \rho(770)$ \\
$m_{K}$ & 496.0 (fit) & $K^{ \pm}(494), K^{0}(498)$ \\
$f_{K}$ & 113.0 (fit) & $K^{+}(113.0)$ \\
$m_{a_{0}}$ & 686.5 & $a_{0}(980), f_{0}(975)$ \\
$m_{K_{0}^{*}}$ & 958.7 & $K_{0}^{*}(1430)$ \\
$m_{\phi}$ & 934.8 & $\phi(1020)$ \\
$m_{a_{1}}$ & 1225.7 & $a_{1}(1260), f_{1}(1285)$ \\
$m_{\eta}\left(\theta=5^{\circ}\right)$ & 546.7 & $\eta(547)$ \\
$f_{\eta}\left(\theta=5^{\circ}\right)$ & 109.3 & $94 \pm 7$ or $91 \pm 6$ \\
$m_{\eta}\left(\theta=0^{\circ}\right)$ & 512.4 & $\eta(547)$ \\
$f_{\eta}\left(\theta=0^{\circ}\right)$ & 106.9 & $94 \pm 7$ or $91 \pm 6$ \\
$m_{K^{*}}$ & 855.4 & $K^{* \pm}(891.6)$ \\
\hline
\end{tabular}

ing these states is that they cannot easily be explained as simple $q-\bar{q}$ bound states (see for instance ref. [11] and references therein).

As pointed out in ref. [8] the relativistic BetheSalpeter formalism does not necessarily disallow scalar or pseudoscalar states with negative charge parity, though these are never observed. Our calculations do not produce any such solutions below $1.2 \mathrm{GeV}$, which we believe is beyond the limit of applicability of the separable approximation in the scalar and pseudoscalar sector.

\section{ACKNOWLEDGMENT}

We are grateful to R. T. Cahill for helpful discussions. The work of CDR was supported by the US Department of Energy, Nuclear Physics Division, under contract number W-31-109-ENG-38.

\section{REFERENCES}

1. C. D. Roberts and A. G. Williams, Prog. Part. and Nucl. Phys. 33 (1994) 475.

2. R. Delbourgo and M. D Scadron, J. Phys. G 5 (1979) 1621.

3. J. Praschifka, C. D. Roberts, R. T. Cahill, Intern. J. Mad. Phys. A 4 (1989) 4929; Y.-b. Dai, C.-s. Huang and D.-s. Liu, Phys. Rev. D 43 (1991) 1717; K.-I. Aoki, T. Kugo and M. G. Mitchard, Phys. Lett. B 266 (1991) 467;
H. J. Munczek and P. Jain, Phys. Rev. D 46 (1992) 438; P. Jain and H. J. Munczek, Phys. Rev. D 48 (1993) 5403; S. J. Stainsby and R. T. Cahill, Mod. Phys. Lett. A 9 (1994) 3551.

4. C. J. Burden, C. D. Roberts and A. G. Williams, Phys. Lett. B 285 (1992) 347.

5. F. T. Hawes, C. D. Roberts and A. G. Williams, Phys. Rev. D 49 (1994) 4683.

6. R. T. Cahill and S. M. Gunner, Quark and gluon propagators from meson data, Flinders University preprint, 1995.

7. C. J. Burden, et al., Spectroscopy of light quark mesons (in preparation).

8. C. H. Llewellyn Smith, Ann. Phys. 53 (1969) 521.

9. C. D. Roberts, Spacelike electromagnetic pion form factor, Argonne preprint ANL-PHY7842-94; C. J. Burden, et. al, Electromagnetic form factors for the charged and neutral kaon (in preparation).

10. Particle Data Group, Phys. Rev. D 50 (1994) 1175. The decay constants given on pages 1443 and 1444 have to be divided by $\sqrt{ } 2$ to compare with the conventions used in this paper.

11. R. Kaminski et al., Phys. Rev. D 50 (1994) 3145 . 


\section{DISCLAMMER}

Portions of this document may be illegible in electronic image products. Images are produced from the best available original document. 


\section{DISCLAIMER}

This report was prepared as an account of work sponsored by an agency of the United States Government. Neither the United States Government nor any agency thereof, nor any of their employees, makes any warranty, express or implied, or assumes any legal liability or responsibility for the accuracy, completeness, or usefulness of any information, apparatus, product, or process disclosed, or represents that its use would not infringe privately owned rights. Reference herein to any specific commercial product, process, or service by trade name, trademark, manufacturer, or otherwise does not necessarily constitute or imply its endorsement, recommendation, or favoring by the United States Government or any agency thereof. The views and opinions of authors expressed herein do not necessarily state or reflect those of the United States Government or any agency thereof. 